\title{
A Inserção do Desenho Paramétrico em Estágios Iniciais do Curso de Arquitetura e Urbanismo a partir do Estudo de Superfícies Curvas
}

\author{
The Insertion of Parametric Design in Initials Stages of Architecture and Urbanism Course \\ Regarding the Study of Curved Surfaces
}

\author{
Luisa Félix Dalla Vecchia \\ UFPel, Brasil \\ luisafelixd@gmail.com \\ Valentina Toaldo Brum \\ UFPel, Brasil \\ valentinatbrum@hotmail.com
}

\author{
Gabriel Martins da Silva \\ UFPel, Brasil \\ gabriel_m_silva@hotmail.com \\ Adriane Borda Almeida da Silva \\ UFPel, Brasil \\ adribord@hotmail.com
}

\begin{abstract}
\section{Introdução}

O desenho paramétrico explora a descrição das relações entre os objetos, estabelecendo interdependências entre diversos elementos. Desta maneira, são geradas variações por meio da transformação e manipulação dos parâmetros, mantendo ainda as condições topológicas das formas (OXMAN, 2006). Para isto, se apoia em técnicas informáticas e, por conveniência para leigos em linguagens textuais, se utiliza de programação visual. Este estudo faz uma reflexão a respeito das consequências da inserção do desenho paramétrico em um processo de ensino/aprendizagem sobre superfícies curvas junto a um contexto específico de formação em arquitetura e urbanismo, cujas linguagens de programação tanto textuais como visuais não fazem parte de conteúdos disciplinares.
\end{abstract}

This paper makes a reflection on the consequences of the insertion of parametric design in teaching / learning processes regarding the study of curved surfaces in the context of training in architecture and urbanism. It shows how this insertion is happening in a discipline on graphic and digital geometry which takes place in the second semester and also the consequences it is bringing to the undergraduate architecture and urbanism course.

Keywords: Parametric Design; Architectural training; Curved Surfaces; Teaching/Learning.

Os conceitos e técnicas de desenho paramétrico vêm sendo tratados sistematicamente na disciplina de Geometria Gráfica e Digital 3 (GGD3), do Departamento de Arquitetura e Urbanismo da Faculdade de Arquitetura e Urbanismo (DAUrb/FAUrb), da Universidade Federal de Pelotas (UFPel), desde o primeiro semestre de 2014. Esta disciplina, de caráter obrigatório, oferecida no segundo semestre do curso, aborda o estudo de superfícies curvas no espaço tridimensional. Contempla uma carga horária de quatro (4) horas semanais. Atualmente, de maneira formalizada na grade curricular, é o único momento em que os estudantes são instrumentalizados a empregar o desenho paramétrico por meio da programação visual.

Como principais motivadores para a inserção do desenho paramétrico neste contexto têm-se: o alto grau de controle e a possibilidade de manipulação da forma, permitidos por tal abordagem, e a explicitação do raciocínio geométrico utilizado, a qual contribui para 0 processo de ensino/aprendizagem específico da disciplina em questão. Os problemas enfrentados no âmbito das ações que levam o grupo docente e discente, envolvido neste trabalho, ao esforço de reestruturar um modo de abordar o ensino de geometria por meio do desenho paramétrico são traduzidos pelo questionamento do quão o referido esforço está provocando a apropriação efetiva para a ação projetual de arquitetura, transcendendo o estudo exclusivo da geometria.

\section{Metodologia}

Este estudo está dividido em cinco etapas:

\section{Revisão Bibliográfica}

A revisão bibliográfica vem sendo permanentemente atualizada, atenta à maneira como o desenho paramétrico vem sendo abordado nas escolas de Arquitetura e Urbanismo do Brasil. Com este propósito foram analisados os anais das edições do SIGraDi de 2012 a 2015, buscando identificar relatos de experiências didáticas brasileiras. Foram identificados oito artigos que disponibilizam dados significativos para este tipo de análise, envolvendo cinco universidades: UFMG, UFMS, UFPel, UFRJ e USP. Dentre estes trabalhos, quatro abordaram o desenho paramétrico sob a forma de workshops (VAZ e CELANI, 2012; BROD, PIRES e BORDA, 2012; LEITE e MARTINS, 2015; PASSARO e HENRIQUES, 2015.), três por meio de disciplinas (STRALEN, BALTAZAR e BERNARDO, 2012; VASCONSELOS et al, 2014; TRAMONTANO, 2015) e um em ambos os formatos (ALVES e TRUJILLO, 2015). Nestes relatos, observou-se que o público alvo são estudantes de 
graduação e somente um deles (LEITE e MARTINS, 2015) abrangendo a extensão.

Quanto ao propósito de uso das técnicas de desenho paramétrico, seis destes casos estão dirigidos diretamente ao desenvolvimento de processos projetuais de arquitetura, enquanto que dois estão focados principalmente no ensino de geometria. Estes dois casos, registrados em 2012 e 2014, referindo-se ao contexto da FAUrb/UFPel, os quais constituiram os antecedentes do que se apresenta neste trabalho.

\section{Inserção do desenho paramétrico na disciplina de GGD3}

A primeira iniciativa de abordagem do desenho paramétrico na disciplina de GGD 3, foi no primeiro semestre de 2012 como parte de um estágio docente de um estudante de mestrado (BROD, 2014). Nesta ocasião foram realizadas atividades de modelagem de obras de arquitetura que envolvessem paraboloides hiperbólicos em sua geometria. Para a caracterização de cada obra específica foi explorada a manipulação dos parâmetros que definem as referidas superfícies e dos procedimentos compositivos aplicados, tais como simetrias, recursões, seções e/ou operações booleanas. Estas atividades ocorreram durante os dois semestres de 2012 e não foram imediatamente incorporadas ao conjunto de atividades da disciplina.

Durante o primeiro semestre de 2014, também como reflexos de estudos na pós-graduação, junto ao Curso de Especialização em Gráfica Digital, foram realizados experimentos com o desenho paramétrico junto à disciplina de Geometria Gráfica e Digital 2 (GGD2), situada no primeiro semestre de formação. Esta é uma disciplina de geometria e representação, do mesmo curso de arquitetura em questão, imediatamente anterior à GGD3. A GGD2 aborda o estudo de formas poliédricas a partir da representação por meio de sistemas de projeção paralelo ortogonal (métodos de projeção cotada e geometria descritiva). A abordagem teve um propósito de introduzir o conceito de desenho paramétrico por meio de jogos, de maneira lúdica, partindose da representação de dobraduras em papel, conforme descrita em VASCONSELOS et al. (2014). Neste contexto os estudantes apenas manipulam parâmetros de um esquema de programação visual previamente configurado. Esta atividade foi incorporada à disciplina desde então.

No segundo semestre de 2014 foi possível inserir sistematicamente exercícios de representação de todas as superfícies tratadas na disciplina de GGD3, não somente ao paraboloide hiperbólico, a partir de técnicas apropriadas para o desenho paramétrico.

Para que isto se efetivasse contou-se com 0 apoio de bolsistas de iniciação científica para o desenvolvimento dos materiais didáticos. Os materiais empregados na disciplina de GGD3, até o primeiro semestre de 2014, abordavam o tema das superfícies curvas através de técnicas de modelagem disponibilizadas por ferramentas como
AutoCAD, 3DsMAX e SketchUp, além do desenho à mão e por instrumentos tradicionais de desenho. Partiu-se da análise destes materiais os quais contemplavam o estudo de treze tipos de superfícies, mantendo-se a sequência didática que vinha sendo adotada na disciplina, buscando apenas contemporizar para abarcar também o desenho paramétrico.

$\mathrm{Na}$ linha superior da Figura 1, exemplifica-se o tipo de material que aborda a modelagem digital a partir da ferramenta SketchUp. Neste tipo são tratadas as regras de geração das superfícies, partindo da definição dos elementos principais, como geratriz e diretriz (imagem da esquerda da figura). No caso do SketchUp, pela limitação da disponibilização de primitivas de programa (formas parametrizadas), os parâmetros que definem estes elementos são atribuídos de maneira independente. E, logo estão submetidos a uma lógica de associação prédeterminada pela própria ferramenta, muitas vezes incompreensível ao projetista. Isto dificulta transformações para além das variações de escala, sem permitir a investigação dos parâmetros da forma, de maneira dinâmica, para realizar ajustes projetuais.

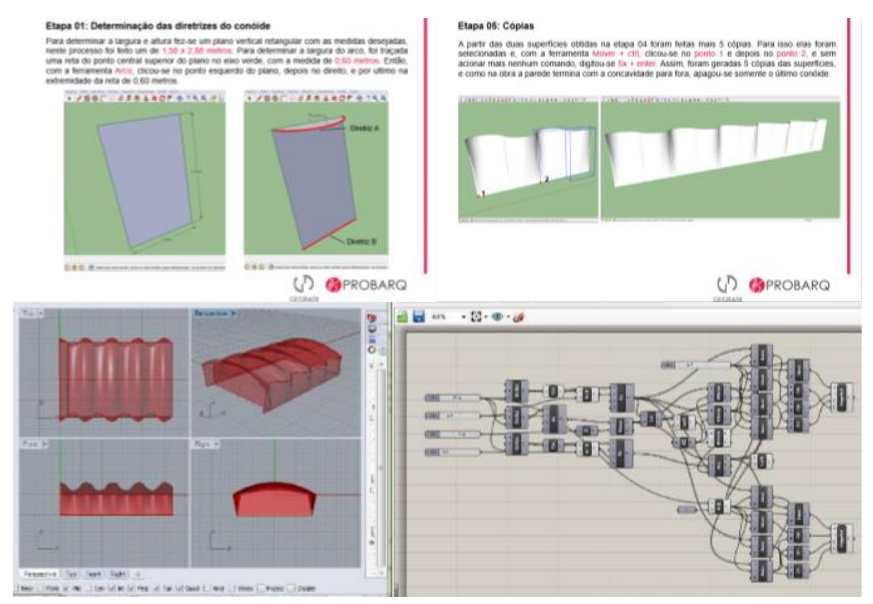

Figura 1: Na linha superior: Recorte de um material didático de apoio à modelagem de superfícies curvas a partir do software SketchUp. Na linha inferior: hipótese de representação paramétrica da Igreja Cristo Obrero de Eládio Dieste.

Fonte: Autores

Os materiais desenvolvidos seguem incluindo a conceituação geométrica de cada tipo de superfície estudada. Entretanto, pela lógica das técnicas de desenho paramétrico as representações figurativas são obtidas de maneira indireta. Tais materiais passaram a explicitar a formulação matemática, para cada uma das treze superfícies abordadas, enfatizando assim os tipos de parâmetros que associam os elementos que as compõe. Além disto, propõem atividades semelhantes àquelas estruturadas em BROD (2014), com os paraboloides hiperbólicos. Com isto, o uso destes materiais tem motivado o desenvolvimento da percepção das variações formais provocadas por transformações de diferentes ordens a partir da manipulação de tais parâmetros pertinentes a todos os tipos abordados na disciplina, adicionados de procedimentos compositivos aplicados a tais superfícies. 
A partir destas atividades agrega-se formalmente à disciplina o objetivo de promover a habilitação dos estudantes ao raciocínio lógico, induzindo-se neste momento por demonstração, por meio do uso do editor gráfico de algoritmos Grasshopper associado ao software Rhinoceros, a compreensão do tipo de abstração matemática envolvida para a representação de cada tipo de superfície. Estas tecnologias empregadas são as mesmas utilizadas nos jogos mencionados anteriormente e também por BROD (2014). Entretanto, a proposta avança em relação aos jogos por seguir a estratégia de BROD (2014), motivando os estudantes a criarem efetivamente a programação visual (seus próprios jogos de controle formal).

Desta maneira, os materiais demonstram como implementar um sistema de representação que permita a alteração dinâmica dos parâmetros e a instantânea alteração da forma. As linhas inferiores da Figura 1 exemplificam um dos materiais desenvolvidos, em que o problema de representação é a compreensão da forma arquitetônica da Igreja Cristo Obrero, do engenheiro uruguaio Eladio Dieste. Neste caso, as formas que lhe dão origem são duas superfícies retilíneas reversas: cilindroides e conóides. Em um primeiro momento um modelo de programação visual de tais formas é exemplificado e acompanhado pelos estudantes que em seguida fazem suas próprias programações para a obra escolhida.

Os materiais buscam promover a associação das mesmas técnicas a outros problemas arquitetônicos de representação. Os estudantes são motivados a identificar outras obras em que poderiam se valer de definições similares, encorajando-os a ampliar o reconhecimento de recursos que resolvam as particularidades de cada obra analisada.

O conjunto de representações da Figura 02 ilustra alguns exercícios realizados pelos estudantes através da programação visual. A imagem na parte superior, à esquerda, se refere ao exercício de estruturar uma programação visual de maneira a garantir concordância entre dois tipos de superfícies regradas, mesmo manipulando parâmetros referentes a cada uma delas (nesse caso conóide e parabolóide hiperbólico). A imagem na parte superior, à direita, se refere à criação de cilindroides e à aplicação de transformações como translação, rotação e reflexão, com garantia de continuidade na superfície final. A imagem à esquerda na parte inferior se refere a um exercício de geração e aplicação de transformações de paraboloides hiperbólicos. Na imagem inferior, à direita, o objetivo foi o de visualizar em tempo real a verdadeira grandeza de seções geradas por um plano manipulado em sua posição no espaço em relação a uma determinada superfície. No caso da ilustração, a posição do plano estava controlada em relação aos raios da geratriz e da diretriz, parâmetros fundamentais para definir uma superfície tórica.

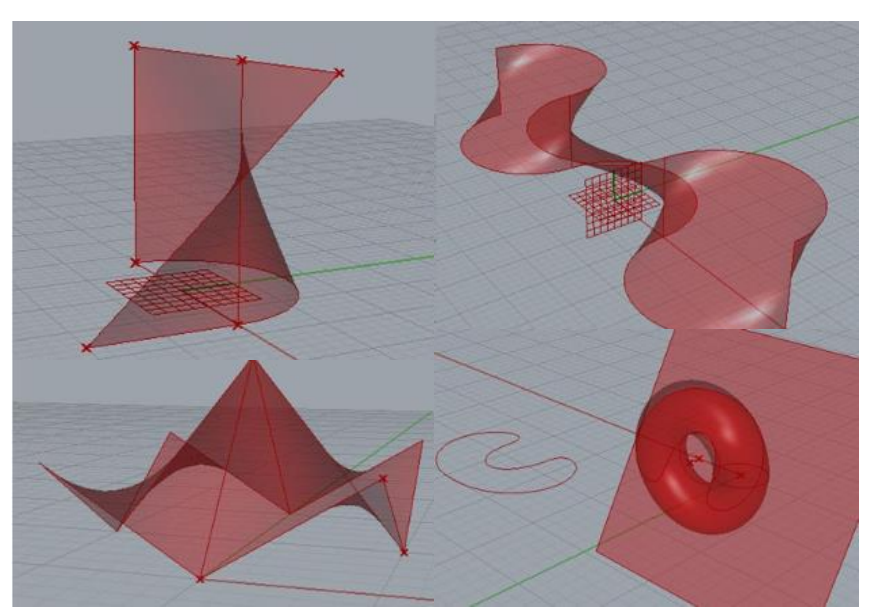

Figura 02: Exercícios realizados pelos estudantes.

Fonte: Autores

\section{A inserção do desenho paramétrico associado à fabricação digital}

A cada semestre as atividades estão sendo revistas buscando um aprimoramento em relação ao que vem sendo feito, especialmente reavaliando as contemporizações (horas aula para cada tipo de atividade).

No primeiro semestre de 2016 buscou-se, além de trabalhar em paralelo com os diferentes meios de representação (a mão, digital e digital através da programação visual), destacar/explorar as potencialidades de cada um em explicitar os parâmetros de controle de cada tipo de superfície abordado. Houve a intenção de que se utilizasse o processo mais adequado para cada uma delas, levando-se em conta que algumas lógicas são similares.

Com esta flexibilização, e consequente ganho de tempo, foi possível trazer para o âmbito da disciplina o investimento na exploração da associação da parametrização aos conceitos de prototipagem rápida e fabricação digital, utilizados experimentalmente ou esporadicamente em momentos anteriores como descritos em PIRES, FÉLIX e BORDA (2016).

As imagens da Figura 03 mostram alguns dos modelos didáticos utilizados para apoiar o estudo dos tipos de seções: sobre superfície cônica e tórica, respectivamente. Estes modelos foram executados por fabricação digital utilizandose de impressão 3D, pelo processo de sobreposição de camadas de material fundido (FDM), neste caso o PLA. Entretanto, a execução destes modelos havia envolvido apenas o corpo docente e da pesquisa. Recentemente a infraestrutura do laboratório de fabricação digital, do contexto em questão, passou de uma para três impressoras 3D, sendo possível então investir na escala de graduação, tendo em vista a disponibilidade também de uma cortadora a laser. As turmas da disciplina estão constituídas por 17 estudantes para 1 professor, trabalhando-se habitualmente com duas 
turmas de maneira integrada em um mesmo horário e espaço de aula.
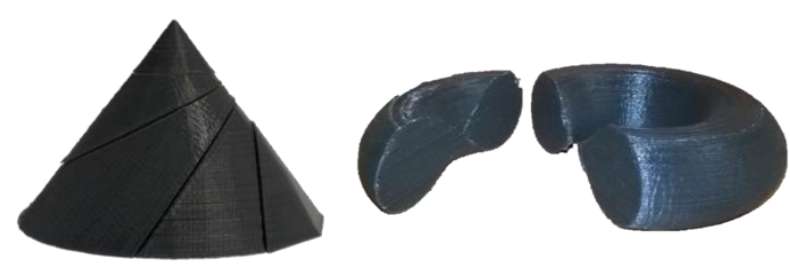

Figura 03: Modelos produzidos para visualização de seções Fonte: Autores

Desta maneira, a partir do primeiro semestre de 2016 a prototipagem rápida passou a estar inserida associada aos processos de parametrização, no âmbito das atividades dos estudantes. Em um primeiro momento foram estudados os conceitos associados à prototipagem rápida e fabricação digital e demonstrados protótipos produzidos por diferentes técnicas. A atividade de aplicação ficou caracterizada, junto ao trabalho final da disciplina, pela análise de uma obra de arquitetura que obrigatoriamente envolvesse uma ou mais das superfícies estudadas no contexto da disciplina. Baseado nas potencialidades e limitações de cada técnica apresentada e levando em conta as possibilidades do contexto desse estudo em termos de disponibilidade dessas tecnologias, cada estudante tem a liberdade de escolher o processo de produção tanto para o modelo digital como para o modelo físico da obra analisada. As Figura 04 e 05 mostram dois exemplos dos modelos físicos produzidos pelos estudantes utilizando-se de processos de corte a laser em papel paraná $2 \mathrm{~mm}$ (figura 04) e impressão 3D em PLA (figura 05). Neste caso, foi selecionado pelo estudante um dos volumes do Centro Cultural Principado de Astúrias de Oscar Niemeyer.

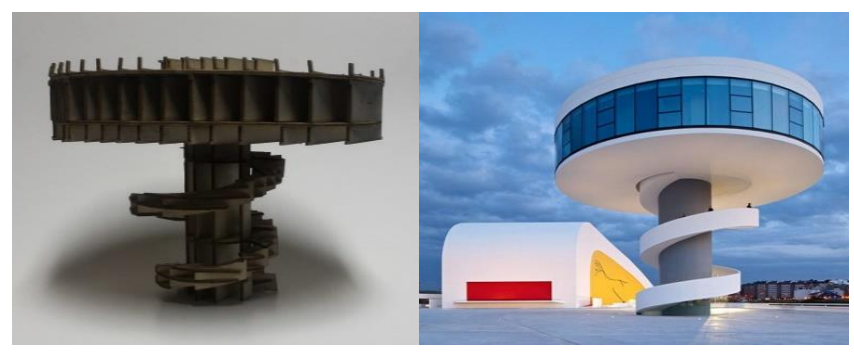

Figura 04: à direita, modelo produzido pelo estudante Arthur Davesac, por meio de corte a laser, referente a um dos elementos do Centro Cultural Principado de Astúrias (à esquerda), de Oscar Niemeyer.

Fonte:Autores

Percebeu-se no primeiro semestre de 2016 um aumento significativo na quantidade de estudantes que optaram por desenvolver os modelos digitais de seus trabalhos finais utilizando-se das técnicas de desenho paramétrico. Subiu de $8 \%$ para $25 \%$, aproximadamente. A figura 05 ilustra mais um dos trabalhos de estudante. À esquerda, acima, a imagem da obra selecionada, do Centro Internacional de Mídia Phoenix do arquiteto Shao Weiping, localizado em Pequim, seguida das imagens dos modelos digitais executados por meio do software Grasshopper associado ao Rhinoceros. E, no canto inferior direito, o modelo físico, impresso em 3D.

Os estudantes ficaram livres também para escolher 0 processo para a produção da maquete. Observou-se que $41 \%$ dos estudantes optou por usar impressão 3D, 46\% optou por utilizar corte a laser e 13\% optou por utilizar técnicas apenas manuais. Cabe destacar que em alguns casos a escolha do processo não foi a mais adequada para expor os aspectos da obra destacados na análise, entretanto, os estudantes se deram conta disso e destacaram esse aspecto em sua apresentação final durante a qual foi realizada uma discussão compartilhando os aprendizados.

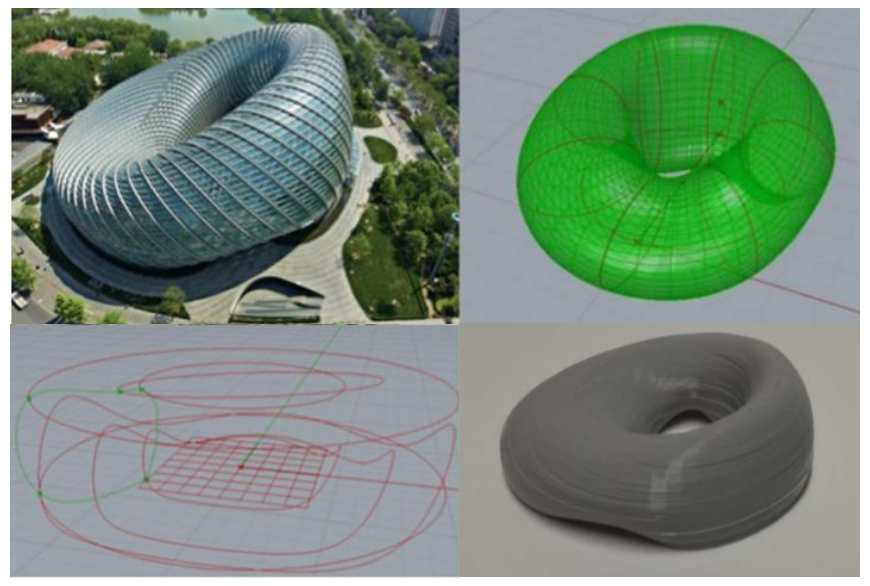

Figura 05: ilustração das representações realizadas pelo estudante Gabriel Lopes referente ao Centro Internacional de Mídia Phoenix do arquiteto Shao Weiping (à esquerda, acima). Na sequencia:imagens dos modelos digitais produzidos por meio do desenho paramétrico e o modelo impresso em 3D em PLA.

Fonte: Autores

\section{Acompanhamento da trajetória dos estudantes}

Vem sendo realizado o acompanhamento da trajetória dos estudantes que cursaram a disciplina de GGD3, após a inserção da abordagem do desenho paramétrico, no que se refere à aplicação da mesma junto às disciplinas de projeto ao longo do curso.

Ainda que seja recente a inserção das técnicas de desenho paramétrico (desde o primeiro semestre de 2014), foram verificados alguns casos pontuais de tentativa de uso de tais conceitos como método projetua. A Figura 06 ilustra um desses casos em que o desenho paramétrico foi usado para manipulação da forma externa de uma capela no contexto de uma disciplina de projeto arquitetônico, que ocorre paralelamente à disciplina de GGD3. Isto ocorreu já no mesmo momento em que foi inserido pela primeira vez (semestre 01/2014). Conforme registrado em Brum, Félix e Borda (2015) destaca-se que houve receio, por parte da estudante, quanto à receptividade dos docentes ministrantes da disciplina de projeto ao uso deste método para a exploração formal. Sendo que, no desenrolar do processo, 
foi compreendida a pertinência de uso para a solução formal adotada. Entretanto, em virtude do ineditismo do método para a disciplina em questão, o processo de orientação foi prejudicado. Isto ficou evidenciado quando se observou a possibilidade de parametrizar também, por exemplo, a forma da porta principal. Junto à solução apresentada ao corpo docente, esta porta não estava associada parametricamente ao plano sobre o qual se localizava, resultando no paralelismo e não na convergência das linhas verticais, como ilustra a maquete da parte superior da Figura 06. Em um momento posterior à conclusão da disciplina, no âmbito da pesquisa, o modelo paramétrico foi manipulado de maneira a implementar a relação de convergência entre este elemento e o plano que o contém, conforme imagem da parte inferior da Figura 06.

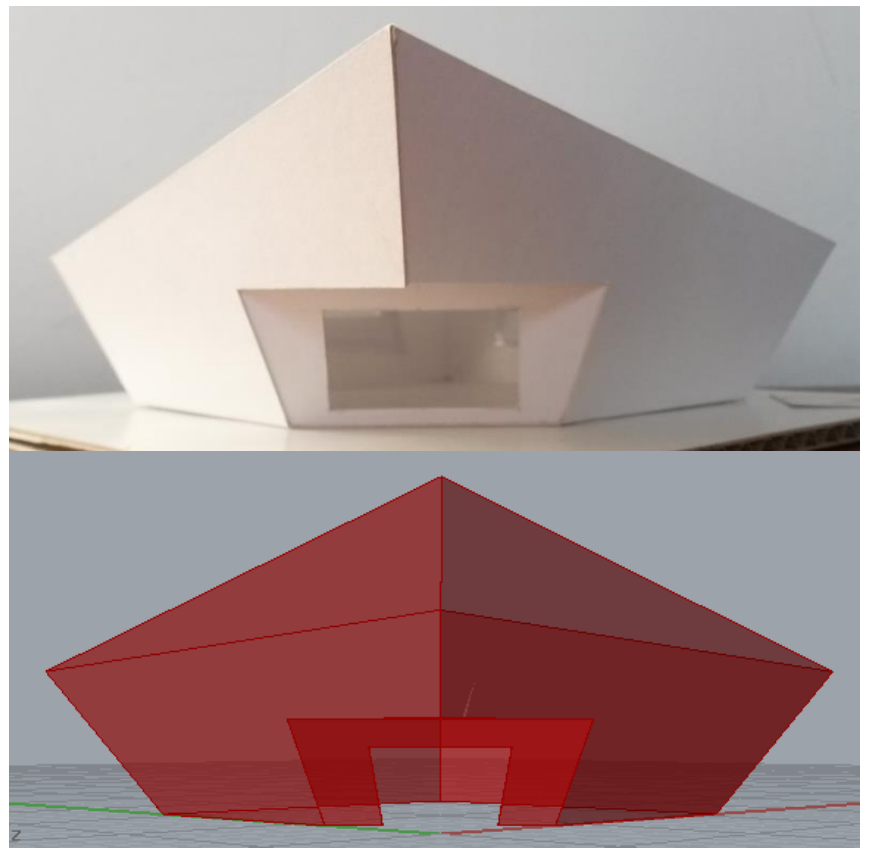

Figura 06: Exercício projetual em que a estudante se utilizou do desenho paramétrico.

Fonte: Autores

Como dados que corroboram para compreender as consequências desta inserção no contexto em questão, pode-se acrescentar o aumento do número de estudantes interessados nos processos de seleção junto ao GEGRADI (Grupo de Estudo em Ensino e Aprendizagem em Gráfica Digital). Este grupo é constituído pelos docentes responsáveis por estas disciplinas de representação junto à FAURB/UFPel. O interesse na apropriação das tecnologias de fabricação digital tem motivado os estudantes, mesmo como voluntários, para dar continuidade ao processo inicializado no âmbito das disciplinas, tendo em vista que ainda em disciplinas de projeto não existe formalizada a continuidade destes estudos aplicados ao projeto de arquitetura. O uso das tecnologias de desenho paramétrico e de fabricação digital no ensino tem motivado o envolvimento dos estudantes em outras atividades, tais como pesquisa e extensão, voltadas a esses tópicos. Era comum junto aos processos seletivos de bolsas termos a proporção de 1/1 ou 2/1 vaga. Tem-se registrado a proporção de 6/1 vaga, mesmo tendo sido ampliado, consideravelmente, o número total de ofertas de bolsas em todas as áreas e nos três segmentos, de pesquisa, ensino e extensão, no âmbito da UFPel.

\section{Análise}

Vem sendo feita a análise sistemática dos resultados a cada semestre para aperfeiçoar a prática didática, especificamente buscando problematizar a experiência, a qual para 0 contexto em questão é inovadora e dependente de uma infraestrutura de corpo docente e tecnológica ainda em formação para o desenho paramétrico. Os dados estão sendo gerados naturalmente junto a um ambiente virtual de aprendizagem (AVA/UFPel) onde os estudantes depositam obrigatoriamente os arquivos, imagens, relatórios e apresentações, em formato digital, constituindo uma memória da dinâmica da disciplina a cada semestre.

\section{Resultados e Discussão}

A abordagem do desenho paramétrico no âmbito da disciplina tem estabelecido, no ambiente de sala de aula, um contexto de aprendizado compartilhado entre professores e estudantes e tem configurado uma atividade permanente de investigação.

Sob o ponto de vista dos docentes envolvidos, atesta-se que a estruturação dos esquemas por meio da programação visual tem exigido uma revisão na abordagem de cada uma das formas estudadas. Exigido um aprofundamento para prévia compreensão e descrição tridimensional da geometria (regras e operações sobre elas), evidenciando a lógica associativa dos modelos figurativos e automatizados aos modelos algébrico-matemáticos, mesmo que de maneira conceitual. Esta abordagem também têm deixado mais explicita a falta de base geométrica e algébrica com que os estudantes chegam à universidade.

Ainda que a inserção esteja em fase inicial, é possível observar, especialmente após à conexão do desenho paramétrico à fabricação digital, maior motivação no que se refere à aprendizagem de superfícies curvas, em relação às transformações e composições geométricas aplicadas sobre elas. E, como demonstrado, já existe o registro de experimentação imediata de uso do desenho paramétrico em disciplinas de projeto.

Considera-se que a inserção do desenho paramétrico na disciplina potencializou e complementou o desenvolvimento das atividades por meios tradicionais de desenho e modelagem digital que já vinham sendo desenvolvidas nesse contexto anteriormente.

Associa-se o aumento na quantidade de estudantes que optaram por desenvolver seus trabalhos utilizando-se das técnicas de desenho paramétrico a uma maior compreensão da própria geometria abordada além do reconhecimento das potencialidades de uso de tais processos. Este aumento se 
deve também ao amadurecimento do corpo docente e da infraestrutura que foi incrementada, dos materiais didáticos e da própria motivação de se ter disponíveis tecnologias de fabricação digital.

Uma das principais dificuldades enfrentadas tem sido a falta de infraestrutura considerada adequada no que se refere a uma sala de aula que contemple tanto o desenho à mão, com pranchetas de desenho, como digital, com computadores.

Considera-se que para estabelecer uma cultura de uso do desenho paramétrico, é importante que ele seja abordado em outros momentos do curso, e não somente em uma disciplina de geometria e representação. E que, além disto, seja integrado ao ensino de maneira multidisciplinar, indo além das questões geométricas e da forma. Para tanto, reafirma-se a importância de estabelecer um diálogo mais efetivo entre as áreas de representação e projeto. Nesta direção, e como principal resultado de toda a experiência, está o movimento que se estabelece junto ao colegiado de Curso da FAURB/UFPel, de reconhecimento da conveniência da apropriação de técnicas de desenho paramétrico junto à prática projetual. Neste momento, encaminha-se uma revisão curricular incluindo uma disciplina de atelier de projeto a ser implementada a partir do primeiro semestre de 2017. Esta disciplina é caracterizada como atelier vertical, permitindo assim uma prática projetual que integra estudantes de diferentes níveis. Entende-se que esta possibilidade promoverá o uso aplicado e imediato dos estudos de geometria como os aqui relatados. Desta maneira, possibilitará a contextualização e extensão da aplicação do desenho paramétrico frente à complexidade da ação projetual, para muito além de uma abordagem geométrica. Associa-se assim, esta consequência, à construção, junto ao contexto em questão, de uma cultura atenta às potencialidades do desenho paramétrico junto à prática de projeto. Com isto, haverá a possibilidade de observar as consequencias em abordar 0 desenho paramétrico em momentos prévios ao projeto, junto às disciplinas de geometria.

\section{Considerações Finais}

Deve-se considerar que as reflexões aqui registradas estão baseadas em resultados imediatos, porém bastante animadores no sentido de criação de uma cultura de reconhecimento das potencialidades das tecnologias de desenho paramétrico para a formação em arquitetura. Estes resultados estimulam o prosseguimento no investimento de inserção destas tecnologias no âmbito das disciplinas de geometria e gráfica digital.

Entretanto, tem-se o receio de que não havendo uma continuidade formativa junto ao contexto de projeto 0 investimento seja em vão para muitos estudantes. Este estudo prosseguirá atento aos desdobramentos após a implementação do atelier vertical que ocorrerá junto à FAURB sob a abordagem de desenho paramétrico junto à formação para a prática projetual de arquitetura.

\section{Agradecimientos}

Este trabalho agradece especialmente à Profa. Dra. Gabriela Celani, parceira do Projeto ACORDA (Análise e Construção de Referenciais Didáticos para Arquitetura: abordagem para o Desenho Paramétrico e Prototipagem Rápida), pela contribuição na formação em desenho paramétrico possibilitada aos professores da disciplina em questão principalmente decorrente das oficinas promovidas pelo LAPAC. Agradece também aos estudantes da disciplina e às instituições UFPel e CNPq, facilitadoras por meio das concessões de bolsas de iniciação científica no âmbito do Projeto ACORDA e PROMETEC (Processo de Ensino e Aprendizagem de Projeto Mediado pelas Tecnologias da Informação e Comunicação em Arquitetura e Design), ambos apoiados pelo edital Universal/CNPq.

\section{Referencias}

Alves, G., Trujillo, J. (2015). Design Cibersemiótico: uma proposta metodológica para o ensino de Processos Digitais de Projeto. XIX Congresso da Sociedade Ibero-americana de Gráfica Digital - SiGraDI 2015, Florianópolis, Brasil.

Brum, V.; Félix, L.; Borda, A. (2015). O uso de técnicas de Desenho Paramétrico junto à prática de projeto no contexto da FAUrb/UFPel: Estudo de Caso. XXIV CIC - Congresso de Iniciação Científica da Universidade Federal de Pelotas. Pelotas, Brasil.

Brod, G., Pires, J., Borda, A. (2012). Um ensaio para inserção do conceito de processos generativos digitais em estágios iniciais da formação em arquitetura. XVI Congresso da Sociedade Iberoamericana de Gráfica Digital - SiGraDI 2012, Fortaleza, Brasil.

Leite, D. P., Martins, J. T. (2015). Processos projetuais emergentes: A utilização de Design Digital e Prototipagem Rápida aplicados em Extensão Universitária. XIX Congresso da Sociedade Iberoamericana de Gráfica Digital - SiGraDI 2015, Florianópolis, Brasil.

Oxman, R (2006). Theory and design in the first digital age. In: Design Studies 27. London: Elsevier.

Passaro, A., Henriques, G. C. (2015). Abrigos Sensíveis, do método ao conceito, superando a instrumentalização. XIX Congresso da Sociedade Ibero-americana de Gráfica Digital - SiGraDI 2015, Florianópolis, Brasil.

Pires, J., Dalla Vecchia, L. F.; Borda, A. (2016). Tecnologias de Representação e Geometria Descritiva: Reflexões sobre um Repertório Docente Dirigido à Arquitetura. Revista Educação Gráfica, vol. 20, ำ1

Tramontano, M. (2015). Quando pesquisa e ensino se conectam: design paramétrico, fabricação digital e projeto de arquitetura. XIX Congresso da Sociedade Ibero-americana de Gráfica Digital - SiGraDI 2015, Florianópolis, Brasil.

Vasconselos, T., Borda, A., Dalla Vecchia, L. F. (2014). A parametrização como experiência prévia para a estruturação de métodos projetuais em arquitetura. XVIII Congresso da Sociedade Ibero-americana de Gráfica Digital - SiGraDI 2012, Montevideo, Uruguai.

Vaz, C. E. V., Celani, M. G. C. (2012). Gramáticas da forma e modelagem paramétrica - uma aplicação na área da arquitetura paisagística. XVI Congresso da Sociedade Ibero-americana de Gráfica Digital - SiGraDI 2012, Fortaleza, Brasil. 